\title{
Switching quantum transport in a three donors silicon fin-field effect transistor
}

\author{
Guillaume Leti, ${ }^{1,2}$ Enrico Prati, ${ }^{1, a)}$ Matteo Belli, ${ }^{1}$ Guido Petretto, ${ }^{1,3}$ Marco Fanciulli, ${ }^{1,3}$ \\ Maud Vinet, ${ }^{4}$ Romain Wacquez, ${ }^{5}$ and Marc Sanquer ${ }^{5}$ \\ ${ }^{1}$ Laboratorio MDM, IMM-CNR, Via Olivetti 2, I-20041 Agrate Brianza, Italy \\ ${ }^{2}$ Ecole Nationale Supérieure de Physique, Electronique et Matériaux, Grenoble Institute of Technology, \\ Grenoble, France \\ ${ }^{3}$ Dipartimento di Scienza dei Materiali, Università degli Studi Milano-Bicocca, Via Cozzi 53, \\ I-20125 Milano, Italy \\ ${ }^{4}$ DRT-LETI-D2NT, 38054 Grenoble, France \\ ${ }^{5}$ SPSMS, UMR-E CEA/UJF-Grenoble 1, INAC, 38054 Grenoble, France
}

(Received 9 September 2011; accepted 23 November 2011; published online 12 December 2011)

\begin{abstract}
We switch the transport along different paths in a system constituted by a phosphorus donor in a silicon quantum dot in complementary metal-oxide-semiconductor technology, coupled with two donors at the source side. The standard Coulomb blockade pattern created by the transport through the $D^{0}$ neutral state of an individual donor located in the channel of the device is modified by two additional randomly diffused nearby donors. By varying the control voltages, the fin-field effect transistor acts as a quantum device which may be used to control alternative current paths through different donors. (C) 2011 American Institute of Physics. [doi:10.1063/1.3669702]
\end{abstract}

We developed a single atom based device in complementary metal-oxide-semiconductor (CMOS) technology which can be tuned among different conductive paths of electrons by virtue of additional donors present in the channel. Atomic scale electronics based on few dopants in the channel of a CMOS device is of major importance for the new generation of Beyond-CMOS devices. ${ }^{1}$ The effects of single dopants in the channel of a silicon device have been characterized in the recent years. ${ }^{2-8}$ The employment of donors in quantum devices has been proposed for their large valley splitting, which is of great importance for quantum information schemes based on the spin of individual electrons. ${ }^{9}$ Here we report on the fabrication of a fin-field effect transistor (FinFET) in CMOS technology with three donors diffused in the channel from the leads and its characterization at cryogenic temperatures. We identified three different conductive paths for sequential tunneling of electrons by comparing the experimental results with numerical simulations which clarify the different role of the three donors. Our investigation represents a proof of principle that it is possible to tune the current path through different single donors in a single quantum device, in view of exploiting quantum degrees of freedom in silicon devices. ${ }^{10}$

The sample is a FinFET produced on a $200 \mathrm{~mm}$ siliconon-insulator (SOI) wafer. The gate electrode has been patterned using electron beam lithography and has a length $L_{G}=30 \mathrm{~nm}$. The size of the channel is width $W_{c h}=50 \mathrm{~nm}$, thickness $T_{c h}=17 \mathrm{~nm}$, and length $L_{c h}=250 \mathrm{~nm}$. Figure 1(a) shows a scanning electron microscope (SEM) image of a sample produced with the exact same procedure, but without back-end process. The wafer is doped with phosphorus, with a density $[P]=10^{18} \mathrm{~cm}^{-3}$.

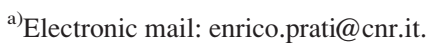

All the measurements were performed at a temperature of $4.2 \mathrm{~K}$, except for the current-voltage characteristics $I_{\mathrm{D}}$ $\left(V_{G}\right)$ which were carried out in the range $4.2 \mathrm{~K}-256 \mathrm{~K}$ for the extraction of the threshold voltage $V_{t h}$. We observed
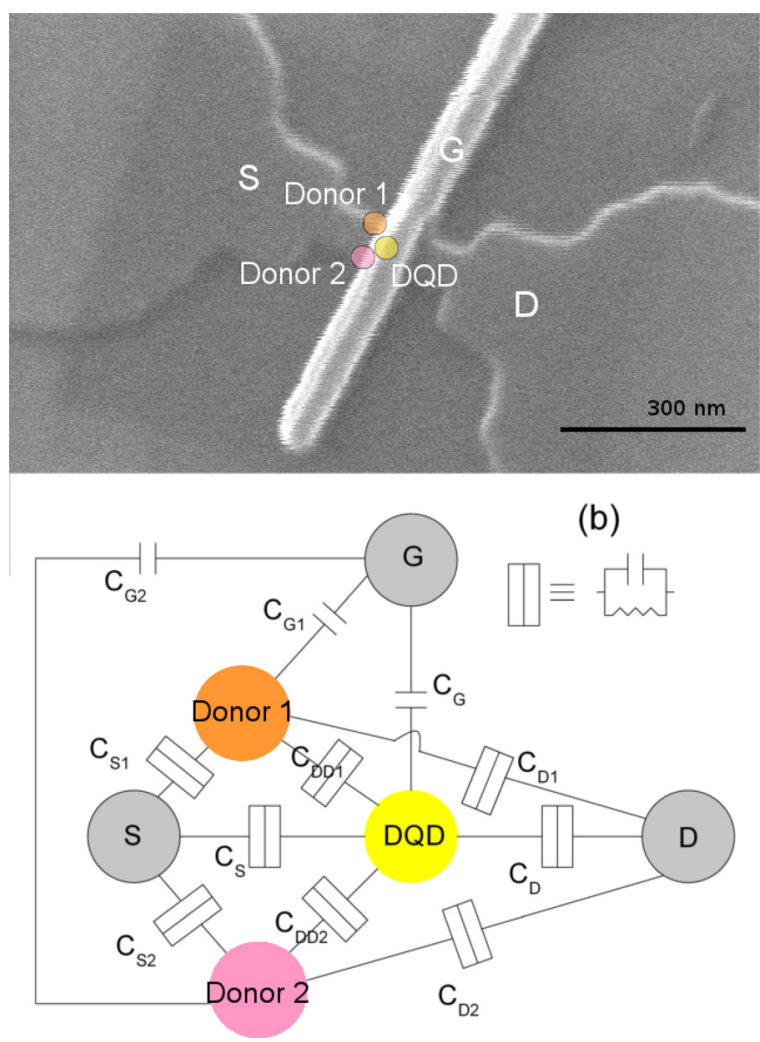

FIG. 1. (Color online) (a) SEM image of the device. For the sake of clarity, the main donor located in the quantum dot (DQD) is pictured in yellow, the donor 1 in orange, and the donor 2 in pink. (b) Electrical model used in the simulations. The DQD is connected to the leads, the gate and the two additional donors. The donors are connected to the leads, the gate, and the DQD. 


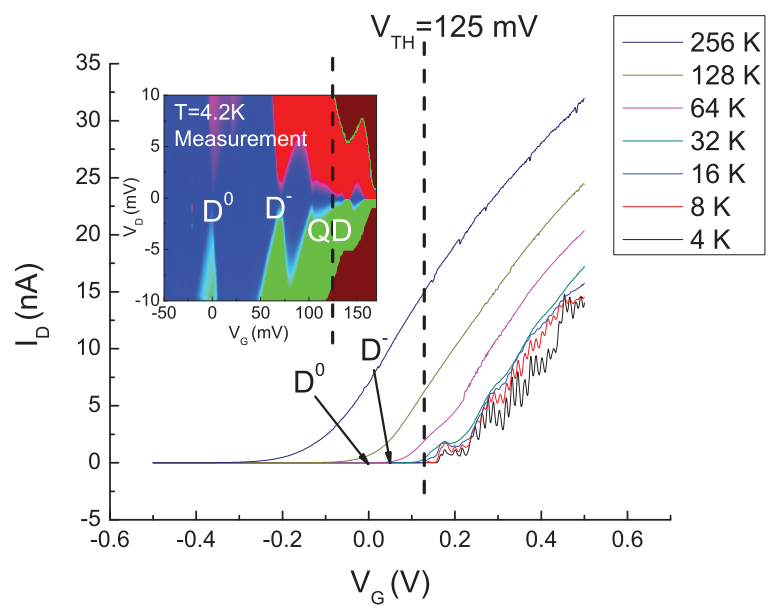

FIG. 2. (Color online) Current-voltage characteristics $I_{\mathrm{D}}\left(V_{G}\right)$ at different temperatures for $V_{D}=1 \mathrm{mV}$. The threshold voltage value is at around $V_{t h}=125 \mathrm{mV}$ (dashed black vertical line). Inset: stability diagram of the device at $4.2 \mathrm{~K}$, whose colorscale span the range $\pm 10 \mathrm{pA}$. The first two peaks of conduction correspond to the $D^{0}$ and $D^{-}$states of the DQD. At high gate voltage value, the conduction occurs by virtue of an electrostatic quantum dot (QD) formed in the channel of the FinFET.

$V_{t h}=125 \mathrm{mV}$ at $4.2 \mathrm{~K}$, as shown in Fig. 2 (the $V_{D}$ value adopted was $1 \mathrm{mV}$ ). The Coulomb blockade of an electrostatic quantum dot is observed below $16 \mathrm{~K}$. The subthreshold stability diagram is shown in the inset of Fig. 2 (with color-
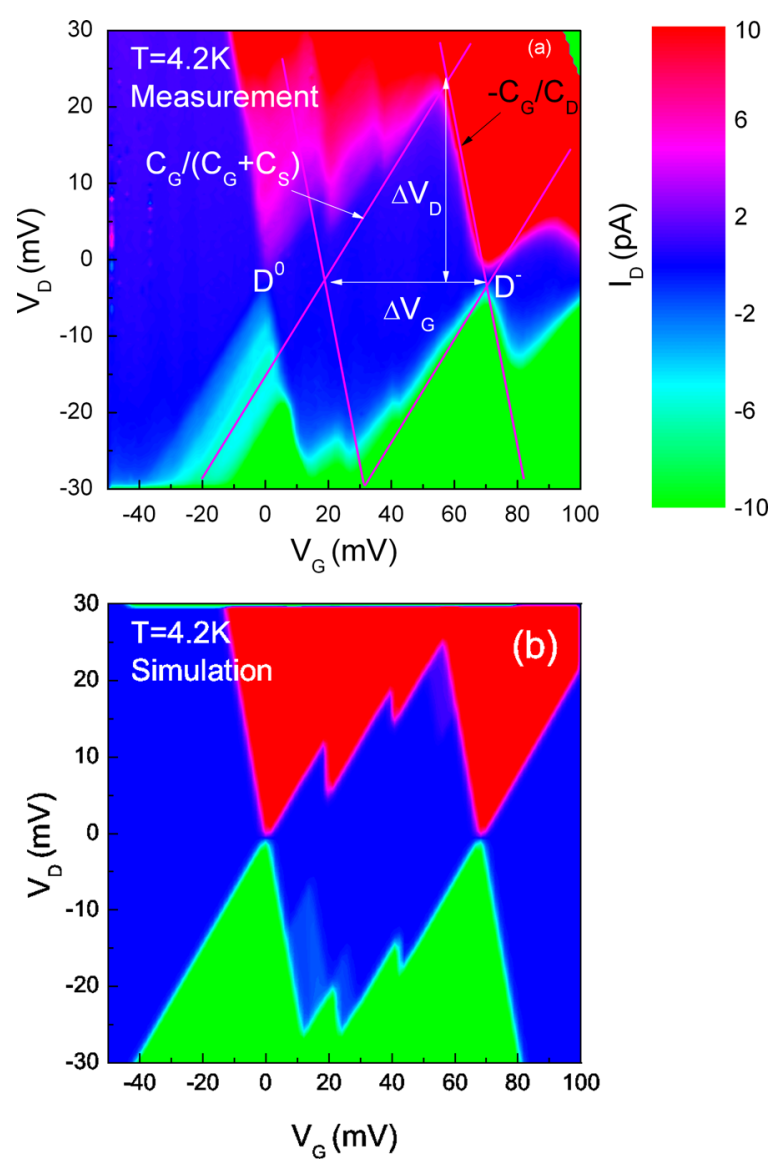

FIG. 3. (Color online) (a) Current stability diagram at $4.2 \mathrm{~K}$ of the FinFET device under investigation. The red solid lines sketch the borders of Coulomb diamonds. (b) Simulated stability diagram of the model outlined in Fig. 1(b).
TABLE I. Comparison of the parameters between measurement and simulation. $C_{s}, C_{d}$, and $C_{g}$ represent the coupling capacitances between the donor quantum dot with source, drain, and gate, respectively, $U$ the DQD charging energy, $C_{d d 1}$ and $C_{d d 2}$ the mutual coupling capacitances between DQD and donor 1 and donor 2 respectively, $\alpha$ is the lever-arm parameter for the DQD and $E_{0}$ represents the energy of DQD ground state with respect to the bottom of the conduction band.

\begin{tabular}{lcc}
\hline \hline & Measurement & Simulation \\
\hline$C_{s}(\mathrm{aF})$ & 1.39 & 1.4 \\
$C_{d}(\mathrm{aF})$ & 1.3 & 1.3 \\
$C_{g}(\mathrm{aF})$ & 2.87 & 2.9 \\
$U(\mathrm{meV})$ & 26.6 & 25.8 \\
$C_{d d 1}(\mathrm{aF})$ & - & 0.2 \\
$C_{d d 2}(\mathrm{aF})$ & - & 0.4 \\
$\alpha(\mathrm{meV} / \mathrm{mV})$ & 0.557 & - \\
$E_{0}(\mathrm{meV})$ & 58.5 & - \\
\hline \hline
\end{tabular}

scale in the range $\pm 10 \mathrm{pA}$ ). By virtue of the different leverarm factors between the two first Coulomb diamonds, also supported by the large addition energy between the first two conductance peaks, they are attributed to the $D^{0}$ and $D^{-}$ states of a phosphorus donor located in the quantum dot (DQD for brevity) randomly present because of the phosphorous background doping of the silicon. ${ }^{4}$ This conclusion is further supported by the comparison with similar samples fabricated with undoped silicon, for which only a single electrostatic quantum dot was observed. All the next peaks are associated to an electrostatic quantum dot formed in the channel of the FinFET. Figure 3(a) shows the stability diagram of the first two conductance peaks, corresponding to the filling of the first two available levels below the conduction band edge of the donor located in the channel. The two portions of the conduction peak inside the first Coulomb diamond are determined by the shifts of the first peak, due to the charging of two donors (called then donor 1 and donor 2) in the proximity of the channel close to the source side. ${ }^{11}$ When full, each of them causes an upward shift of the energy levels of the dot, hence the presence of current in a region normally blocked. From this stability diagram we were able to extract the capacitive coupling of the main donor with the gate $C_{G}$, the drain $C_{D}$, and the source $C_{S}{ }^{12}$ We extracted the ground state energy $E_{0}=58.5 \mathrm{meV}$ from the conduction band edge and the lever-arm factor $\alpha$, yielding a charging energy value $U=26.6 \mathrm{meV}$. Both $E_{0}$ and $U$ are consistent with previous results of donors in similar samples. ${ }^{2,4,6-8}$ All these values and those used in the simulation are listed in Table I.

The simulations have been performed with a constant interaction model and the current is calculated from the

TABLE II. Parameters of the two donors used in the simulation. $C_{s(i)}, C_{d(i)}$, $C_{g(i)}, C_{d d(i)}$ represent the coupling capacitances of either donor $1(i=1)$ or donor $2(i=2)$ with respectively source, drain, gate, and DQD, as specified in Fig. 1(b).

\begin{tabular}{lllcc}
\hline \hline & $C_{s(i)}$ & $C_{d(i)}$ & $C_{g(i)}$ & $C_{d d(i)}$ \\
\hline Donor 1 $(\mathrm{aF})$ & 0.08 & 0.005 & 1 & 0.4 \\
Donor 2 $(\mathrm{aF})$ & 0.05 & 0.01 & 1.2 & 0.6 \\
\hline \hline
\end{tabular}



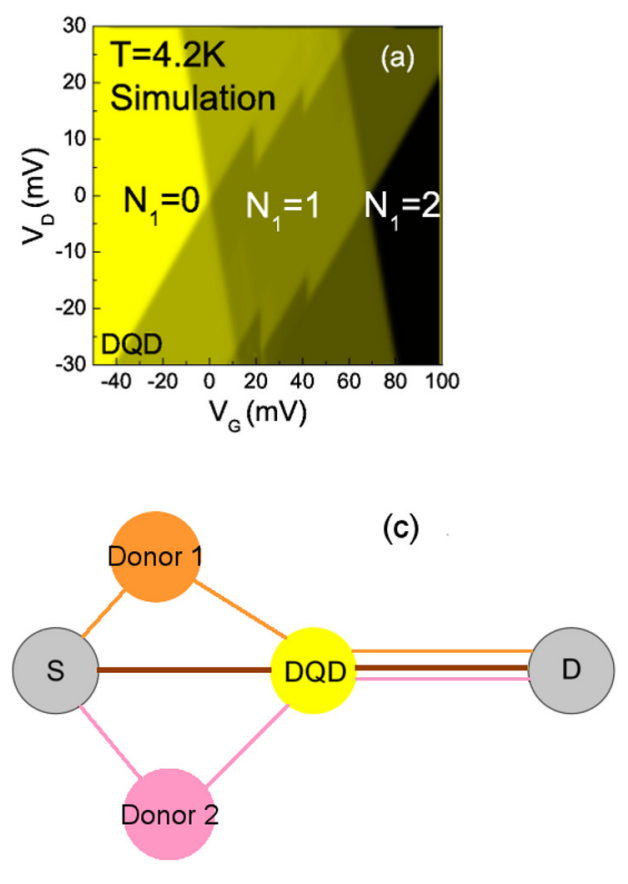
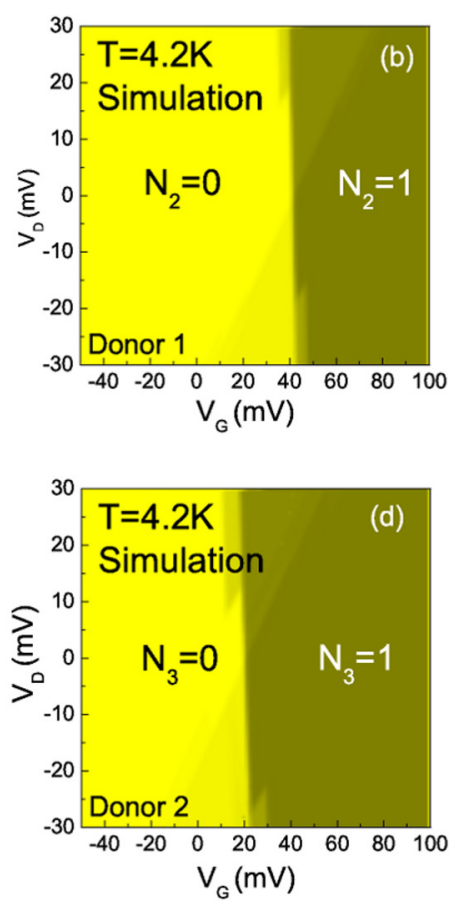

FIG. 4. (Color online) (a), (b), and (d) Occupation probability respectively in DQD, donor 1 and donor 2; (c) the different electron paths in the device. stationary solutions of a transition rate equation. ${ }^{13}$ The current value is normalized to a maximum conductance set as an input empirical parameter. The model is shown in Fig. 1(b), and consists of three donors. The DQD localized in the center of the channel has a double spin degenerate ground state. The energy separation between the corresponding conductance peaks on the stability diagram represents the charging energy $U$. The two other donors are localized close enough to the main donor and to the source to have a significant effect on the stability diagram, but they are weakly coupled to the drain. They are both coupled to the dot, but there is no coupling between the two donors. All the parameters of the two donors used for the simulation are listed in Table II. The simulations were performed at a temperature of $4.2 \mathrm{~K}$. The electrostatic quantum dot has been excluded in the simulations for the sake of simplicity, as its effect occurs at voltages higher than those interesting in the analysis. For the sake of completeness we mention that we simulated the stability diagrams corresponding to several other types of topologies and couplings, though none of them were as satisfactory as the one in Fig. 3(b) in reproducing key features of the measured stability diagram.

The results obtained from the simulations reproduce accurately the experimental stability diagram as shown in Fig. 3(b). Figures 4(a), 4(b) and 4(d) show the occupation probabilities inside the three dots, allowing us to determine the electron filling sequence inside the device $\left(N_{1}, N_{2}\right.$, and $N_{3}$ being the number of electrons, respectively, in the main donor DQD, the donor 1, and the donor 2). The simulation allows to extract the current flowing through the single branches depicted in Fig. 4(c) (not shown) and hence to determine the conductivity path in all the areas of the stability diagram. Above the first conductance peak, the $D^{0}$ state of the main donor is occupied by one electron so current is blocked. For $V_{G}=20 \mathrm{mV}$, an electron enters the donor 2, shifting up the energy level of the DQD. The shift allows a flow of current if the bias window is high enough, in an area which, without the donor 2, would be blocked. The current flows through the main donor from the leads, but there is also a very small contribution from the donor 2. This effect is more visible at positive bias as it is coupled more strongly to the source than to the drain, leading to a lower probability for the electrons to enter at negative bias. The third peak at $V_{G}=40 \mathrm{mV}$ is explained by the same process, involving the donor 1 . The three paths available for the electrons are shown in Fig. 4(c). The second Coulomb blockade peak is associated to the $D^{-}$state of the main donor. According to the different biasing conditions, the device is tuned from the unperturbed path (brown line in Fig. 4(c)) at low bias voltage to the modified path through donor 1 (orange) and 2 (pink) at higher bias.

To conclude, we fabricated a device based on the passage through an individual donor located in its channel in CMOS technology, with two extra donors in the proximity of the source. Such extra donors provide alternative conduction paths to current flow. Such a tunable quantum device constitutes a proof of principle of discrimination of different conductive paths generated by individual donors, with possible applications in Beyond-CMOS devices.

This work has been supported by the EU-FP7 project AFSiD (Grant No. 214989).

${ }^{1}$ M. Pierre, R. Wacquez, B. Roche, X. Jehl, M. Sanquer, M. Vinet, E. Prati, M. Belli, and M. Fanciulli, Appl. Phys. Lett. 95, 242107 (2009).

${ }^{2}$ M. Pierre, R. Wacquez, X. Jehl, M. Sanquer, M. Vinet, and O. Cueto, Nat. Nanotechnol. 5, 133 (2010).

${ }^{3}$ M. Hori, T. Shinada, Y. Ono, A. Komatsubara, K. Kumagai, T. Tanii, T. Endoh, and I. Ohdomari, Appl. Phys. Lett. 99, 062103 (2011).

${ }^{4}$ E. Prati, M. Belli, S. Cocco, G. Petretto, and M. Fanciulli, Appl. Phys. Lett. 98, 053109 (2011).

${ }^{5}$ D. Moraru, A. Udhiarto, M. Anwar, R. Nowak, R. Jablonski, E. Hamid, J. C. Tarido, T. Mizuno, and M. Tabe, Nanoscale Res. Lett. 6, 479 (2011). 
${ }^{6}$ G. P. Lansbergen, R. Rahman, C. J. Wellard, I. Woo, J. Caro, N. Collaert, S. Biesemans, G. Klimeck, L. C. L. Hollenberg, and S. Rogge, Nat. Phys. 4, 656 (2008).

${ }^{7}$ E. Prati, R. Latempa, and M. Fanciulli, Phys. Rev. B 80, 165331 (2009).

${ }^{8}$ K. Y. Tan, K. W. Chan, M. Möttönen, A. Morello, C. Yang, J. van Donkelaar, A. Alves, J.-M. Pirkkalainen, D. N. Jamieson, R. G. Clark, and A. S. Dzurak, Nano Lett. 10, 11 (2010).

${ }^{9}$ M. Friesen, Phys. Rev. Lett. 94, 186403 (2005).
${ }^{10}$ J. Verduijn, G. C. Tettamanzi, G. P. Lansbergen, N. Collaert, S. Biesemans, and S. Rogge, Appl. Phys. Lett. 96, 072110 (2010).

${ }^{11}$ H. Huebl, C. D. Nugroho, A. Morello, C. C. Escott, M. A. Eriksson, C. Yang, D. N. Jamieson, R. G. Clark, and A. S. Dzurak, Phys. Rev. B 81, 235318 (2010).

${ }^{12}$ S. M. Sze and K. K. Ng, Physics of Semiconductor Devices, 3rd ed. (Wiley, New York, 2007), p. 360.

${ }^{13}$ M. Pierre, M. Hofheinz, X. Jehl, M. Sanquer, G. Molas, M. Vinet, and S. Deleonibus, Eur. Phys. J. B 70, 475 (2009). 
Applied Physics Letters is copyrighted by the American Institute of Physics (AIP). Redistribution of journal material is subject to the AIP online journal license and/or AIP copyright. For more information, see http://ojps.aip.org/aplo/aplcr.jsp 